\section{La mutación pragmática de la crítica de arquitectura}

Josep Maria Montaner
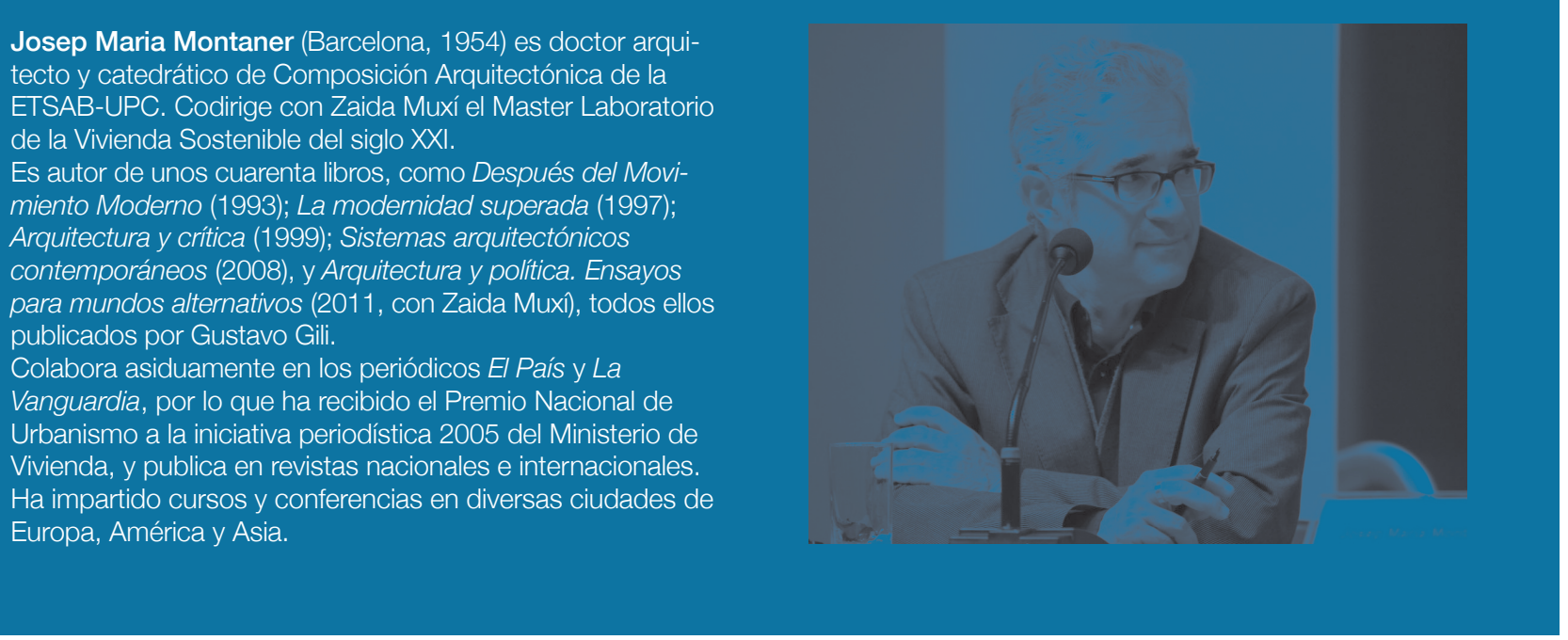

- n las últimas décadas se está produciendo una fuerte transformación de las coordenadas de la teoría y la crítica de esta transformación, intentando establecer una cartografía fiable en este mundo confuso y atomizado.

\section{Postcrítica}

Es evidente que entre los años sesenta y ochenta del siglo pasado se produjo el periodo dorado de la teoría y crítica de la arquitectura y el urbanismo contemporáneos, fundacional de la crítica al movimiento moderno, con las aportaciones de figuras como Manfredo Tafuri, Carlo Aymonino, Aldo Rossi, Jane Jacobs, Alison y Peter Smithson, Robert Venturi, Denise Scott Brown, Colin Rowe o Kenneth Frampton, yendo desde el espectro más radical y marxista hasta el más formalista, nostálgico y defensor de la autonomía formal de la arquitectura.

En la medida en que este periodo ya forma parte de la historia, la situación actual de transformación puede valorarse en dos sentidos. De manera optimista, entendiendo que no se trata de una crisis total sino de una mutación, que conlleva la aparición de nuevas interpretaciones de la crítica que superarán esta fase fundacional contemporánea. O de manera nostálgica, echando en falta los valores que tuvo este periodo crítico y sosteniendo que se ha pasado, simplificando, de la trascendencia del mar-
xismo y el estructuralismo a la levedad del pragmatismo.

En la línea nostálgica se sitúa el libro póstumo de Martin Pawley, La extraña muerte de la crítica de arquitectura (2007), que toma el título de uno de sus últimos ensayos, poniendo en sintonía su
propia decadencia con la de la crítica de arquitectura. Pawley fue uno de los críticos británicos de la segunda mitad del siglo XX, junto a Reyner Banham y J.M. Richards. De todas formas, su título expresa un hecho impactante e inquietante: la crítica
de arquitectura, tan influyente hace pocas décadas, en los últide arquitectura, tan influyente hace pocas
mos años tiene un papel poco relevante.

\section{Crítica y crisis}

En la actualidad podemos establecer que en el caso de la crítica de arquitectura se suman dos crisis culturales: la general y la propia.

Este proceso se enmarca en el fenómeno más general de la pérdida de papel representativo de la intelectualidad progresista que, aunque exista, tiene menos posibilidades de expresarse In los medios dominantes que hace unos años. El mundo de Internet ha potenciado una "realidad expandida", con innumerables protagonistas, y ya no hay referentes míticos, como
otras épocas, sino que el panorama es totalmente abierto.

En el terreno de la arquitectura, este proceso de mutación se percibe más profundamente, al compararla con este periodo del proyecto crítico y al ser totalmente necesario un replanteamiento de criterios frente a los cambios estructurales. Ello se produce, además, en un contexto en el que el saber de la arquitectura ha perdido el papel decisorio y de liderazgo que tuvo en el período de entreguerras y en la postguerra. Entonces las políticas de vivienda y equipamientos fueron inspiradas, en gran parte, por arquitectos, y sentaron las bases del estado del bienestar. Hoy la arquitectura y el urbanismo tienden a ser serviles a los objetivos financieros e inmobiliarios. A este descrédito ha contribuido la complicidad con la especulación, la corrupción y el alarde de poder. Y ello obliga a este cambio total.

\section{Pragmatismo}

En estas condiciones, los rasgos que distinguen a las nuevas generaciones de críticos tienen que ver con el pragmatismo y la operatividad. Se ha consolidado un giro copernicano en relación a algunas características de la teoría y la crítica de los años sesenta y setenta: de los teórico-teóricos, como Manfredo Tafuri o Colin Rowe, se ha pasado al predominio de los teórico-prácticos, como Peter Eisenman, Rafael Moneo y Rem Koolhaas, aunque iniciaran su carrera como teóricos.

El pragmatismo en filosofía es, históricamente, una corriente esencialmente norteamericana-Charles Sanders Pierce, William James o John Dewey - . Sin embargo, tiene múltiples interpre-
taciones. No lo entendemos hoy como el pragmatismo poco reflexivo, antintelectualista y ajeno a la planificación y sistematicidad, liderado por la cultura norteamericana, sino como un pragmatismo basado en la experiencia y abocado a la acción, que sabe aprender del conocimiento teórico. Recientemente ha habido las aportaciones del filósofo norteamericano John Rajcknan, que desde 1997 habla de "pragmatismo diagramático" y del libro recopilatorio de William S. Sanders, The New Architectural Pragmatism, (2007) dando la palabra a actores
como Alejandro Zaera-Polo, Robert Somol, Sarah Whiting y James Corner.

Quisiéramos que este pragmatismo enlazara con lo que reclamaba el filósofo y psicoanalista Félix Guattari. En sus escritos -varios de ellos realizados con Gilles Deleuze-, Guattari buscaba una "nueva pragmática" y para ello propuso la "ecosofía" como nueva praxis. La ecosofía comporta una visión holística que exige una radical transformación interrelacionando tres niveles: el del medio ambiente; el de lo social e interpersonal; y el mental, de una nueva estructura del sujeto.

La operatividad tiene la vertiente optimista de reclamar un activismo sistemático y vital, superando el mandamiento político y moral de Manfredo Tafuri, quien se oponía radicalmente a la "crítica operativa". Tafuri insistió en que ésta siempre legitima las producciones arquitectónicas y urbanas del capitalismo, y no puede evitar contribuir a su ideología. La crítica contemporánea se ha liberado de esta cautela y, aunque siga siendo manipulada y sirva de justificación al capitalismo, las condiciones han cambiado.

\section{Características del cambio}

Podemos recapitular y señalar algunas características interrelacionadas que diferencian buena parte de la teoría y crítica contemporáneas en relación a este momento fundacional de los años sesenta y setenta.

Una: la "operatividad" es una exigencia presente en los escritos contemporáneos. Hoy no es entendible una posición que tema la utilización ideológica de lo escrito, aunque esta instru-

Dos: son mucho menos reconocidos los críticos que actúan sólo desde la crítica, los que se dedican exclusivamente a la investigación, que los arquitectos profesionales, que escriben además de proyectar y construir obras reconocidas. Aquí queda un trabajo pendiente de la crítica para reclamar la revaloriconstituye un modo de transmisión del conocimiento sino que revitaliza la investigación, la acción social, la defensa del pa-
trimonio, la promoción de colectivos, el refuerzo de las redes. Tres: una parte de los textos contemporáneos, hechos desde un trabajo profesional que necesita de la promoción pública y privada para poder existir,
prometido de la crítica.

Cuatro: Ios escritos raramente parten de cuestiones conceptuales o teóricas, sino desde la obras, empíricamente. Los tuales o teoricas, sino desde la obras, empiricamente. Los desde la imagen y no desde el razonamiento. Véase, en este sentido, la línea de publicaciones iniciada por Rem Koolhaas con su Delirious New York y seguida por MVRDV o BIG.

Un síntoma de estas posiciones pragmáticas y operativas es el papel tan relevante que se otorga a Peter Zumthor, cuya cualificada obra se basa en la construcción y la materialidad, ceptuales y sociales muy conservadores y nostálgicos, negando las referencias utilizadas y donde todo parece resultado de una experiencia subjetiva. Más que antes, la obra construida se ha convertido en la referencia legitimadora y ya no lo es e proyecto experimental.

Hoy queda lejana la teorización de Emil Kaufmann sobre la revolución de la arquitectura a partir de los arquitectos de la llustración, como Boullée, Ledoux y Lequeu, que dibujaron mucho y construyeron poco; o son menos relevantes las disquisiciones de Tafuri sobre el papel tan representativo de Gian Batista Piranesi, del que se conocen más sus grabados que sus pocas y magníficas obras realizadas. mentalización se produzca. zación de su aportación, demostrando que la crítica no solo pero que en sus escritos expresa unos planteamientos con-
Diversidad de posiciones

En este recorrido sobre la condición actual de la crítica se pueden establecer diversas posiciones, reconociendo previamente la influencia de teóricos pertenecientes a una generación similosofía del Arte y la Arquitectura en la Yale University y que ha legitimado los principios conceptuales de la postmodernidad en arquitectura; Juhani Pallasmaa (1936), que con su defensa de una arquitectura que intensificara todos los sentidos intenta reinventar al individuo contemporáneo, aislado y que percibe, que no está en sociedad; y Dalibor Vesely (1034), que ha (1934), que ha planteado una crítica conceptual a las insuficiencias en la evolución partiendo de la fenomenología y la hermenéutica.

Se puede establecer una cierta polarización de posiciones: las que siguen siendo críticas y las que se han desarrollado en este terreno de una postcrítica neoliberal. Y dentro de cada uno de los polos las posiciones también se subdividen.

En la continuidad del proyecto crítico tenemos a los representantes de una posición más académica, como Michael Hays, de raíz marxista y "tafuriana", quien dirigió Assemblage (19861990); Beatriz Colomina (1952), que arrancó de estas posiciones, pero que ha ido hacia una mirada mas procapitalista y que es autora de libros clave sobre los media o la sexualidad de (1972) que en su texto The Project of Autonomy. Politics and Architecture within and against Capitalism (2008) ha iniciado la recuperación y reinterpretación del proyecto crítico dentro y contra el capitalismo en los años miento de los "autónomos italianos", como Tronti, Cacciari, Negri, Tafuri y Rossi, argumentando que en aquel período la defensa de la autonomía tenía que ver con la argumentación de la "autonomía obrera" como proyecto cultural y político.

En esta misma continuidad, pero en un ámbito más alternativo e hipercrítico, en la línea del urbanismo radical, como el marxista David Harvey o el anarquista Mike Davis (1946), estaría la figura de Dolores Hayden, que defiende la visión feminista y la historia de las propuestas de vida comunitaria frente al sprawl y la contaminación del territorio.

Por otra parte, en la línea liberal también se daría una posición más académica, la postcrítica heredera del formalismo analítico de Colin Rowe, Peter Eisenman, Tony Vidler y Rem Koolhaas, que está representada, especialmente, por Robert Somol y su énfasis en los diagramas.

En la línea productivista se sitúan los neopragmáticos o tecnoadministradores, que en España están representados por Alejandro Zaera-Polo, Federico Soriano o el grupo Metápolis. Su objetivo ha sido legitimar los argumentos de la Administración y hacerse un hueco en los encargos.

En Europa no aumentan los focos de la crítica, más allá del grupo editorial de la revista Lotus en Milán, con Pier Luigi Nicolin a la cabeza, y de facultades de arquitectura como la de la Delft University of Technology, la facultad de Arquitectura en la Universidad Católica de Lovaina, con Hilde Heynen,
Londres, que continúa su tradición vanguardista.

En el caso de Cataluña, las dificultades de la teoría y la crítica son recurrentes. Algunos autores se han incluido a sí mismos en sus escritos, como Oriol Bohigas o Helio Piñón; y el gran teórico que tuvimos, Ignasi de Solà-Morales, es reconocido pero no por el peso de su propuesta crítica, de alcance internacional. Hoy intenta dominar la ambición de los arquitectos profesionalistas, que han creado sus agencias, lobbies y blogs para medrar repartiendo placebo de crítica y copar el espacio de la curadoría.

Sin embargo, no existe otra manera posible de conceptualizar, proponer alternativas y replantear la arquitectura si no es desde la responsabilidad, la mirada a largo término y el esfuerzo de la teoría y la crítica. En este sentido, es un hecho positivo visible que la intelectualidad barcelonesa haya sabido desarrollar un pensamiento urbano, que surge de la interpretación de la rea-
lidad, estableciendo teorizaciones a partir del aprendizaje de la práctica y siendo capaz de interactuar.

\section{Crítica e investigación}

El predominio del pragmatismo y la omnipresencia del proyecto arquitectónico han llevado en los últimos años a la afirmación en falso de que el mismo proceso de diseño es un proceso de investigación y creación de conocimiento; que proyectando se está teorizando. Lo argumentan Alejandro Zaera-Polo, Philippe Starck y Robert Somol en el libro editado por Sanders, otorgando la primacía al proyecto arquitectónico por encima de la teoría.

Recientemente ha sido el arquitecto, teórico y activista Jeremy Till quien ha contestado a lo que él considera el tercer mito sobre la investigación arquitectónica. El primer mito sería que la arquitectura es autónoma; el segundo que la arquitectura no es arquitectura, no es suficiente por sí misma y ha de buscar su legitimación en la autoridad de otras disciplinas; y el tercer mito argumenta que construir un edificio es investigacion. Til el edificio por sí solo nunca habla sino que exige todo un trabajo de investigación: requiere todo un análisis de los procesos del proyecto, de los significados y, sobre todo, de los usos y de la vroyecto, de los significados y, sobre todo, de los usos y de implica una buena investigación, sino que la investigación tiene que ver con este análisis y conceptualización profundos sobre 


\section{Lamarck y las indexadas}

\section{Ignacio Paricio}

"A medida que los individuos ..... cambian de hábito ....reciben
influencias que cambian poco a poco las proporciones de sus
partes ... sus facultades y hasta su misma organización" partes ... sus facultades y hasta su misr

E I profesor Tremendo Curriculum se despertó apoyado en - su mesa de trabajo. Se había quedado dormido sobre un papel en blanco donde debía plasmar sus nuevas ideas, las líneas de investigación para el año que empezaba. Tenía un dolor de cabeza notable. Desde hacía un tiempo notaba un extraño bulto en nada. Increíblemente el famoso investigador estaba seco.

Inquieto, se volvió hacia el montoncillo de artículos a publicar. La especialista Indexación Garantías se los había dejado listos para enviar. ¡Qué chica mas maja! Calculó rápidamente los puntos que enviar. ¡Qué chica mas maja! Calculo rápidamente los puntos que dos y ahora debidos. Sonrió ante el numero de créditos que se le iban a conceder. Entró en el software en el que se contabilizan sus "puntos" como investigador y sonrió. Una oleada de placer recorrió su mente reconociendo la facilidad con la que se movía en esa laberíntica trama inquisitorial que tantas dificultades le había planlaberíntica trama inquisitorial que tantas dificultades le había planni la única manera de evaluar su trabajo. El bulto en el temporal se activó y pareció crecer mientras calculaba.

Se volvió hacia el papel en blanco para retomar la orientación de los proyectos de futuro, para intentar de nuevo activar su reconocida creatividad. Nada, estaba vacío. El dolor de cabeza se cambió al lado derecho. Curiosamente tenía la sensación de que ese temporal se deprimía. iiDios mío!! ¿Es que no se le iba a ocurrir nada que impulsase la línea de investigación que le había hecho famoso unos años atrás?

Sus colaboradores le esperaban para la reunión del grupo de investigación. Todos ellos necesitaban esas ideas que debían alimentar sus curriculums. Por cierto, que Trilero Investigador estaba pidiendo otro sexenio. Se le estaba acercando peligrosamente en pidiendo otro sexenio. Se le estaba acercando peligrosamente en
los puntos. No había problema, tenía recursos. Dulcemente, suavemente se activó el hemisferio izquierdo. Ya no dolía, era agradable recordar que solo tenía que llamar a su profesora asociada dable recordar que solo tenía que llamar a su pro
Creatividad Gestora para solucionar el problema.

No eran malos sus chicos. Quizás un poco demasiado ambiciosos y focalizados en la evaluación cuantitativa. Sobre todo esa chica del este, Suma Puntos. No tenía pudor. Pero bueno, mientras siguiese colocándole en todos sus trabajos como coautor... Aunque a veces eran insignificantes ... todo suma, señorita Suma.

\section{La implantación extensiva y aditiva como método histórico de intervención en arquitectura}

\section{Arcadi Pla i Masmiquel}

\begin{abstract}
a arquitectura debe tener potencialidades híbridas; en el preL sente momento histórico, lleno de revisiones y recapacitaciones, se hace más necesaria que nunca la capacidad de evolución, adición y crecimiento de los edificios, tan natural y propia del pasado. En contra de arquitecturas cerradas en sí mismas, que no aceptan la evolución sociologica de costumbres y necesidades de los usuarios ni la adaptación a las nuevas necesidades y costes eneracumulación de sistemas y tipos, puede ser mas adecuada.
\end{abstract}

En este sentido, afrontamos la restauración de la rectoría de la Capilla románica de Sant Martí de Solamal en el Valle de Bianya, Capilla románica de Sant Martí de Solamal en el Valle de Bianya, en la comarca gerundense de La Garrotxa, más como una suma unitaria y cerrada en su uso.

En las edificaciones rurales y masías tradicionales era habitual la construcción de cuerpos añadidos para ampliar las instalaciones, cada uno de ellos ejecutado con los medios y tecnologías constructivas que correspondian a cada época; en ese sentido es importante subrayar la idea que de que solo se interviene en lo construido a partir de lo contemporáneo.

La ermita de Sant Martí de Solamal se entiende a través de las diversas transformaciones que ha recibido, desde el siglo X, siendo una capilla de una sola nave hasta el siglo XII, su ampliación con dos naves laterales en el XVI y, finalmente, con la adicion de la sacristía (XVIII) y la rectoría (XIX). De esta manera no es una edificación, sino un conjunto que se enriquece con los elementos de la explotación agraria como el pajar cubierto o las zonas de trabajo.

El proyecto, debido a esos valores acumulados por la historia del lugar, no se plantea como una reforma sino como un plan global de restitución de la historia del lugar y su adaptación a las nuevas necesidades de este momento. Para cada actuación se delimita la estrategia constructiva y compositiva, aportando un conjunto
Incompetente. Demasiadas historias en común. Por suerte se le había ocurido una solución brillante. La universidad acababa de aprobar el reconocimiento como investigación de las horas de gestión. Menos mal, eso cerraba el círculo virtuoso: tenemos que hacer mucha gestión para permanecer a flote en este mundo investigador, por lo tanto es lógico que se reconozca esa dedicación administrativa como méritos investigadores y capacidad creativa. $Y$ ese es el futuro de mi inefable Aceptado.

Dios mío, jaún tenía que hojear la tesis de esa chica vasca, Enkuaderna Kualkierkosa! Por cierto ... ¿a quién iba a poner en el tribunal de una cosa tan flojita? ¡Ah sí, claro, a aquella italiana tan mona..! Cómo se llamaba ... Mañana Pormí.

Sus ojos se volvieron hacia su lectura abandonada hace unos meses. Sí, aquello de Lamarck: la función crea el órgano. No recordaba muy bien por qué lo estaba leyendo, pero sintió que le había afectado. Apartó de su mente esos pensamientos negativos. Se encontraba bien. No sentía ninguna necesidad de ahondar en esas inquietudes.

¡Qué raro! Se estaba palpando la cabeza y, aunque ya no le dolía, su temporal derecho parecía hundido.

PD.- Después de redactar la fábula anterior, leo en el País (14/06/13) el artículo de Holm-Detlev Köhler a propósito de la entrega del último premio Príncipe de Asturias a la socióloga Saskia Sassen. "La tercera enseñanza de Saskia Sassen nos lleva a la guido ningún mas importantes de nuestrá expoca no ha consede nuestras agencias de evaluación, que anteponen siempre el mismo criterio: tres publicaciones JCR (Journal Citation Reports) mismo criterio: tres publicaciones JCR (Journal Citation Reports) en los últimos cinco años. Sassen no tiene ni una, sino que ha publicado libros e informes, fruto de proyectos de investigación de verdad y referencias fundamentales para académicos compromesion, etc., pero se ha resistido a la práctica de inflar su currículum cír articulos estandarizados sin interés ni lectores, más allá de circulos de amigos do citación mutua. Estamos entregando nuestra calidad científica a Thompson Reuters (la empresa gestora de los JCRs) igual que la calificación de nuestras economías a Fitch, Moody's y Standard \& Poor's. La estandarización de nuestra enseñanza universitaria y de nuestra producción científica nos llevará a universidades sin debates, investigaciones sin compromiso y un sistema académico sin pensamiento.

Ignacio Paricio es Doctor arquitecto y catedrático del Departamento de Composición de la E.T.S.A.Barcelona. futura quiebra de nuestro sistema académico-universitario. Una de

la obra. Por último, la clave es que el conocimiento ha de ser comunicable y muchas veces los arquitectos pragmáticos, por muy buenas obras que realicen, no son capaces de comunicar procesos, significados y usos.

Y aquí aparece otra cuestión clave: la transmisión de conocimiento a través de los nuevos medios, especialmente en Internet. Por una parte, estas redes de comunicación están cambiando los modos y ritmos de transmisión del saber pero, por otra, la gran variedad hace que unas tengan

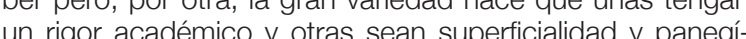
rico. ¿Cómo introducir en estos medios las pautas de los métodos de investigación y transmisión del saber, y cómo metodos de investigación y transmisión del saber, y cómo incorporar los valores de lo que se edita en estos medios en los criterios de valoración académicos? Visibilizando en qué medida han tenido capacidad de cambiar las coordenadas y recurriendo a expertos en estos nuevos formatos que
aporten criterios críticos equiparables a los que ya tienen las aporten criterios críticos equiparaciones convencionales.

\section{Por una teoría pragmática}

Este contexto reclama que las nuevas teorías partan de otras coordenadas: rechazo a la pretendida autonomía de la forma arquitectónica en relación a lo político y social; sintonía con todo tipo de medio de comunicación y con las posibilidades de las industrias locales; incorporación de as nuevos modos de trabajo colectivo y de cooperacion. Todo ello se desarrolla en el futuro libro Del diagrama a las experiencias, hacia una arquitectura de la acción. Mientras abstracto de los diagramas, ha de enraizarse en la vida y la experiencia, reencontrándose con la vertiente activista y experimental que tuvo en otros momentos.

Este proceso de transformación, altamente esperanzador, está caracterizado por este pragmatismo que huye de teorías previas e intenta aprender de la experiencia. La insistencia en la pragmática es un rasgo positivo y está relacionada cia en la pragmática es un rasgo positivo y está relacionada cado losis de realismo, intervenir en realidades concretas. De la lógica productivista e impositiva de la prefabricación y de los aprioris platónie impositiva de la prefabricación y de los aprioris platónicos de la crítica tipológica se ha pasado a la rehabilitación, a operaciones versátiles de menor tamaño y a una mayor capacidad de encaje en el contexto y de creación de vida vecinal. Véanse, como ejemplo, los libros que explican las intervenciones de la ONG Architecture for Humanity escritos por sus fundadores, Cameron Sinclair y Kate Stohr, marcados por este optimismo de la estrategia, la pragmática y la operatividad: esta arquitectura de cooperación se va aprenfuerte base teórica inicial lo que les lanzó a crear el colectivo y la actividad.

Es en este panorama de mutación que las propuestas auténticas relacionadas con el activismo social han podido avanzar: ONGs, grupos activistas, colectivos, experiencias de cooperación, etc. En cambio, la esfera académica tiene de cooperacion, etc. En cambio, la esfera academica tiene encerrada en su mundo de élite, de autosatisfacerse con encerrada en su mundo de elite, de autosatisfacerse con dicalidad de un proyecto crítico. Un riesgo que es posible dicalidad de un proyecto crítico. Un riesgo que es posible la construcción de interpretaciones y propuestas en relación a la sociedad y a la praxis; con la construcción de una nueva relación dialéctica entre la teoría y la práctica.

ARCHITECTURE FOR HUMANITY (Ed.) Design Like You Give a Damm (2).
Building Change from the Ground Up, Abrams, New York, 2012.

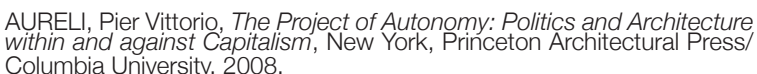
Columbia University, 2008

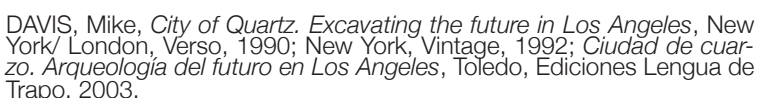

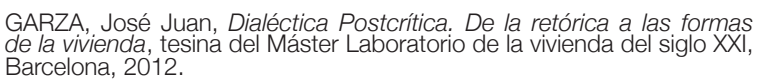

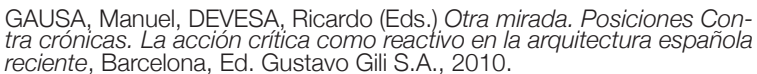
GUATTARI, Félix, Las tres ecologías, Valencia, Pre-Textos, 1990. HARRIES, Karsten, The Ethical Function of Architecture. Cambridge,
Mass., The MIT Press, 1997 . HAYDEN, Dolores, The Power of Place. Urban Landscapes as Public
History, Cambridge, Mass., The MIT Press, 1995 . MARTínEZ, Francisco José, Hacia una era post-mediática. Ontología,
politica y ecología en la obra de Félix Guattari, Mataró/Barcelona, Mon-
tesinos, 2008. MONTANER, Josep Maria, Arquitectura y critica, Ed. Gustavo Gili S.A.,
Barcelona, 1999 (22 edición revisada y ampliada, 2007); Arquitetura ex
critica, Barcelona, Ed. Gustavo Gili S.A., 2007. que no renuncia a la modernidad en los cuerpos modernos pero respetando la idiosincrasia de cada elemento en particular.

Renuncia, pues, a la construcción integrada y compacta, proponiendo una edificación dispersa de las diferentes partes, empezando por la más importante y significativa, que no es otra que la de despegarse de la construcción mas genuina (la lglesia) proponiendo una nueva relación de diálogo con el entorno más inmediato en base a una multipropuesta híbrida con soluciones diversas adaptadas a las necesidades y tipos de restauración o de nueva construcción para cada caso. Lo dicho, una propuesta híbrida en las formas, en las estrategias de cada caso, en los sistemas constructivos que califican cada época.

Arcadi Pla i Masmiquel es arquitecto y profesor de Proyectos en la E.T.S.A.Barcelona y en La Salle-Universitat Ramon Llull.
MONTANER, Josep Maria, Arquitectura y crítica en Latinoamérica, Bue-
nOS Aires, Ed. Nobuko, 2011. PALLASMAA, Juhani, Los ojos de la piel, Barcelona, Ed. Gustavo Gili,
S.A., 2006. PAWLEY, Martin, The strange death of architectural criticism. Collected
writings, London, black dog publishing, 2007 ROWE, Colin, KOETTER, Fred, Ciudad Collage, Barcelona, Ed. Gustavo
Gili S.A., 1981, 1998 . SAUNDERS, William S. (Ed.) The New Architectural pragmatism, Univer-
sity of Minnesota Press, Minneapolis/London, 2007. SOLÀ-MORALES, Ignasi de, Diferencias. Topografía de la arquitectura
contemporánea. Barcelona, Ed. Gustavo Gili S.A., 1995 .

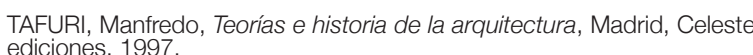
VÉSELY, Dalivor. Architecture in the Age of Divided Representation: The
Question of Creativity in the Shadow Of Production, Cambridge, Mass.
The MIT Press, 2004. ZUMTHOR, Peter, Atmósferas, Barcelona, Ed. Gustavo Gili S.A., 2006. 